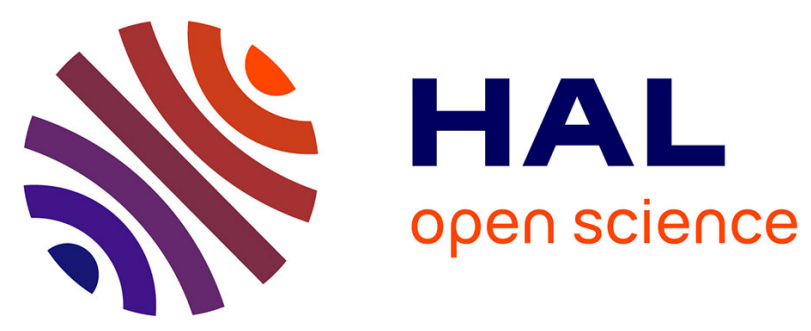

\title{
Development and comparison of high accuracy thermal ionization methods for uranium isotope ratios determination in nuclear fuel
}

\author{
Alexandre Quemet, A. Ruas, V. Dalier, C. Rivier
}

\section{To cite this version:}

Alexandre Quemet, A. Ruas, V. Dalier, C. Rivier. Development and comparison of high accuracy thermal ionization methods for uranium isotope ratios determination in nuclear fuel. International Journal of Mass Spectrometry, 2019, 438, 10.1016/j.ijms.2019.01.008 • cea-02339918

\section{HAL Id: cea-02339918 https://hal-cea.archives-ouvertes.fr/cea-02339918}

Submitted on 21 Oct 2021

HAL is a multi-disciplinary open access archive for the deposit and dissemination of scientific research documents, whether they are published or not. The documents may come from teaching and research institutions in France or abroad, or from public or private research centers.
L'archive ouverte pluridisciplinaire HAL, est destinée au dépôt et à la diffusion de documents scientifiques de niveau recherche, publiés ou non, émanant des établissements d'enseignement et de recherche français ou étrangers, des laboratoires publics ou privés.

\section{다)(1) $(5$}

Distributed under a Creative Commons Attribution - NonCommerciall 4.0 International 
1 Names of the authors: Alexandre Quemet ${ }^{1}$, Alexandre Ruas ${ }^{2}$, Vincent Dalier ${ }^{1}$ and Cédric

2 Rivier $^{1}$

3 Title: Development and comparison of high accuracy thermal ionization mass

4 spectrometry methods for uranium isotope ratios determination in nuclear fuel

5 Affiliation(s) and address(es) of the author(s):

$6-{ }^{1}$ CEA, Nuclear Energy Division, Research Department of Mining and Fuel 7 Recycling Processes, F-30207 Bagnols sur Cèze, France

$8-{ }^{2}$ Onsite Laboratory Team, Nuclear Material Laboratory, Office of Safeguards 9 Analytical Services, Department of Safeguards, International Atomic Energy 10 Agency, Tokyo Regional Office, Seibunkan Bldg, 9F, 1-5-9 Iidabashi, Chiyoda11 ku, Tokyo 102-0072, Japan

12 E-mail address of the corresponding author: alexandre.quemet@ cea.fr 
14 Development and comparison of high accuracy thermal

${ }^{2}$ Onsite Laboratory Team, Nuclear Material Laboratory, Office of Safeguards Analytical Services, Department of Safeguards, International Atomic Energy Agency, Tokyo Regional Office, Seibunkan Bldg, 9F, 1-5-9 Iidabashi, Chiyoda-ku, Tokyo 102-0072,

\section{Keywords}

34 TIMS; minor isotope ratio; multi-dynamic sequence; total evaporation method 


\section{Introduction}

37 Uranium is the most essential element of the nuclear fuel cycle. It is present at different

38 steps with different isotope composition: in uranium mine (natural uranium), in the

39 enrichment process (depleted and enriched uranium), in the fuel fabrication (enriched

40 uranium), in power reactor and in the reprocessing process (reprocessed uranium).

41 Accurate measurements for uranium isotope and concentration are necessary in the

42 nuclear field [1-3]. Knowing the isotope composition and uranium concentration is also

43 of prime interest for safeguards and forensics purposes: the ${ }^{235} \mathrm{U}$ isotope abundance

44 indicates the enrichment level of the nuclear material, the ${ }^{234} \mathrm{U}$ isotope abundance

45 determination provides information on the material origin and finally, the ${ }^{236} \mathrm{U}$ isotope is

46 a marker of uranium origin (natural, fallout from nuclear test or accident) $[1,2,4,5]$.

47 One of the reference techniques for the isotope ratio measurement is the Thermal

48 Ionization Mass Spectrometry (TIMS) [6]. Two TIMS measurement methods are

49 commonly used: the classical and the total evaporation method [6-8]. In the classical

50 method, the different isotopes are collected in a limited period of the sample evaporation

51 and the isotope ratios are mathematically corrected of the isotope fractionation. Isotope

52 fractionation comes from an evaporation difference between the light and the heavy

53 isotopes, causing a bias on measured isotope ratios. In the total evaporation method (TE

54 method), the isotopes are collected during the entire sample evaporation. Thus, this

55 method is barely affected by the isotope fractionation and is a reference technique for

56 major isotope ratio determinations like ${ }^{235} \mathrm{U}^{238} \mathrm{U}[1,2,7]$.

57 The analyses of the ${ }^{234} \mathrm{U} /{ }^{238} \mathrm{U}$ and ${ }^{236} \mathrm{U} /{ }^{238} \mathrm{U}$ isotope ratios can be more complicated. First,

58 weak signals close to the detection limit make accurate measurements difficult. The most

59 commonly used detector for isotopic analysis by TIMS is the Faraday cup coupled to a

$6010^{11} \Omega$ current amplifier. This detector is highly stable helping reaching a high accuracy

61 measurement (i.e. measurement trueness and precision). However, this detection system

62 is not adapted for weak signals. The development of the $10^{12}$ and $10^{13} \Omega$ current

63 amplifiers helps improving the Faraday cup sensitivity [1,9]. When the isotope

64 abundance becomes even lower, it is necessary to use other types of detectors such as the 
65 Secondary Electron Multiplier (SEM). The SEM improves dramatically the TIMS 66 sensitivity [1,2]. However, the low stability of the SEM makes low uncertainty 67 measurements difficult [1]. The abundance sensitivity is another cause of bias for the 68 minor isotope ratios measurement: it is the contribution of the major isotope peak tail (i.e. $69{ }^{235} \mathrm{U}$ or ${ }^{238} \mathrm{U}$ ) to the minor isotope detection (i.e. ${ }^{234} \mathrm{U}$ or ${ }^{236} \mathrm{U}$ ). The retardation filter 70 associated with the SEM decreases the abundance sensitivity by 2 orders of magnitude, 71 improving the measurement bias [1,2]. It is also possible to correct the abundance 72 sensitivity with a mathematical correction. In that case, different measurement sequences 73 can be dedicated to the abundance sensitivity measurement. Also, abundance sensitivity 74 measurement requires the use of the classical method. Another possibility to overcome 75 the isotope fractionation and correct the peak tailing is the Modified Total Evaporation 76 method (MTE). This method consists in interrupting the total evaporation process 77 regularly to perform corrections and signal optimization [10]. This method has the 78 benefits of both the total evaporation method to overcome the isotope fractionation and 79 the classical method to apply corrections using several sequences.

80 ATALANTE is a nuclear facility of the French Alternative Energies and Atomic Energy 81 Commission dedicated to research on the spent nuclear fuel reprocessing process and the 82 management of long-lived radioactive waste. The ATALANTE analysis laboratory is 83 devoted to elemental, isotopic and physico-chemical analyses and nuclear measurements 84 applied to samples of medium and high activity for the ATALANTE R\&D programs. In 85 order to evaluate the laboratory performances and to guarantee the result reliability for 86 the uranium isotope ratio and concentration determination in diverse physico-chemical 87 forms such as pellets or dissolution solutions, the laboratory participates to different 88 Round Robin Test (RRT). The present study focuses on the "2017 Nuclear Material 89 Round Robin" organized by the International Atomic Energy Agency (IAEA) which aims 90 at determining the uranium isotope ratio and mass fraction in nuclear materials. The total 91 evaporation method using different detectors and the classical method using multi92 dynamic sequences for the minor isotope ratios measurement are compared in terms of 93 accuracy, simplicity of use and analysis duration. 
97 All solutions were prepared using polypropylene flasks, except for the uranium solutions,

98 which were prepared in PFA vials. $3 \mathrm{~mol} \mathrm{~L}^{-1}$ and $8 \mathrm{~mol} \mathrm{~L}^{-1}$ nitric acid solutions were 99 prepared by diluting high purity nitric acid (Merck, Suprapur) with deionized water 100 (resistivity: 18.2 M $\Omega . c m$ ). A high precision scale (Mettler-Toledo, WXTP 205) was used 101 to prepare all solutions. Weighings were repeated at least twice.

102 Analytical method validation for the uranium isotope determination was performed on 103 the U015 Certified Reference Material (CRM) provided by the National Institute of 104 Standard and Technology (NIST). The isotope composition of this CRM and the RRT 105 sample are similar. This solution is certified for the ${ }^{234} U /{ }^{238} U(0.00008634(92), k=2)$, $106{ }^{235} \mathrm{U} /{ }^{238} \mathrm{U}(0.015565(16), \mathrm{k}=2)$ and ${ }^{236} \mathrm{U} /{ }^{238} \mathrm{U}(0.0001666(10), \mathrm{k}=2)$ isotope ratios.

108 Each participant of the "2017 Nuclear Material Round Robin" received a uranium oxide 109 pellet $\left(\mathrm{UO}_{2}\right)$ of about $5 \mathrm{~g}$ in a $20 \mathrm{~mL}$ HDPE vial (hereafter referred to as RTT sample). 110 The RTT sample is a uranium fuel pellet fabricated in Brazil with a uranium isotope 111 composition close to a low enrichment uranium oxide pellet before irradiation in 112 Pressurized Water Reactor [11]. The TIMS requires working with liquid samples. Thus, 113 the first preparation step was the dissolution of the pellet. It was weighed and about $11415 \mathrm{~mL}$ of $8 \mathrm{~mol} \mathrm{~L}^{-1}$ nitric acid was added. This solution (pellet + nitric acid) was heated 115 at $135^{\circ} \mathrm{C}$ in a PFA vial until complete dissolution. The pellet dissolution solution 116 (hereafter referred to as RRT solution), which had a uranium concentration about $117250 \mu \mathrm{g} \mu \mathrm{L}^{-1}$, was diluted with $3 \mathrm{~mol} \mathrm{~L}^{-1}$ in order to obtain solutions of concentration 118 suitable for isotopic analysis: about $4 \mu \mathrm{g} \mu \mathrm{L}^{-1}$ and $1 \mu \mathrm{g} \mu \mathrm{L}^{-1}$. 
121 The Thermo Fisher Triton TIMS used for the experiments and the deposit technique were 122 previously described in detail [12]. The TIMS is equipped with 9 Faraday cups (all are 123 movable except the central denoted $\mathrm{C}$ ) which can be coupled to $10^{11} \Omega$ current amplifiers 124 (9 are available and hereafter Faraday cups coupled with $10^{11} \Omega$ amplifiers are referred to 125 as FC 11) or a $10^{12} \Omega$ current amplifier ( 1 is available and hereafter a Faraday cup 126 coupled with a $10^{12} \Omega$ amplifier is referred to as FC 12). 4 Faraday cups are positioned in 127 low masses (noted L1 to L4) and 4 Faraday cups are positioned in high masses (noted H1 128 to H4). The TIMS is also equipped with one fixed discrete dynode Secondary Electron 129 Multiplier located behind the central Faraday cup (hereafter referred to as SEM). The 130 SEM is combined with a high abundance filter (RPQ for Retarding Potential 131 Quadrupole). The SEM calibration was performed using the method described in [12].

132 Isotopic analysis methods

\section{The total evaporation method}

134 The TE method applied in the present study was described in details in previous work for 135 the ${ }^{235} \mathrm{U} /{ }^{238} \mathrm{U}$ major isotope ratio measurement $[1,2,8,12]$. The quantity of uranium 136 deposit was $1 \mu \mathrm{g}$. This uranium amount allows the accurate determination of the $137{ }^{235} \mathrm{U} /{ }^{238} \mathrm{U}$ isotope ratio in compliance with the International Target Value (ITV) [1,2]. The $138{ }^{238} \mathrm{U}^{+}$ion beam target intensity was measured using the FC 11 exclusively that was fixed 139 at $15 \mathrm{~V}$ whereas the ${ }^{235} \mathrm{U}^{+}$ion beam was measured either by FC 11 or FC 12.

140 For the method validation, 6 measurements were performed using the FC 11 and the 141 U015 CRM. Afterwards, for the RRT sample, the ${ }^{235} \mathrm{U} /{ }^{238} \mathrm{U}$ isotope ratio analyses were 142 performed using different detector configurations: 5 analyses were performed using the 143 FC 11 to collect ${ }^{235} \mathrm{U}$ and ${ }^{238} \mathrm{U}$ isotopes, and 5 analyses were performed using the FC 12 144 to collect ${ }^{235} \mathrm{U}$ and the FC 11 to collect ${ }^{238} \mathrm{U}$. In fine the reported isotope ratio was the 145 average of all the measurements. 
146 The ${ }^{234} \mathrm{U} /{ }^{238} \mathrm{U}$ and ${ }^{236} \mathrm{U} /{ }^{238} \mathrm{U}$ isotope ratios were also acquired on the U015 CRM with the

147 TE method at the same time as the ${ }^{235} \mathrm{U} /{ }^{238} \mathrm{U}$ isotope ratio measurements. 3 analyses were 148 performed using the FC 12 to collect the ${ }^{234} \mathrm{U}$ isotope and the SEM to collect the ${ }^{236} \mathrm{U}$ 149 isotope and 3 analyses were performed using the FC 12 to collect the ${ }^{236} \mathrm{U}$ isotope and the 150 SEM to collect the ${ }^{234} \mathrm{U}$ isotope.

152 The TE method is an accurate and simple analytical method for major isotope ratio 153 measurements (typically ${ }^{235} \mathrm{U} /{ }^{238} \mathrm{U}$ isotope ratio) [7]. However, the TE method has 154 limitations for the minor isotope ratios measurements because these measurements 155 require other corrections such as: the SEM calibration, changes in the peak centering and 156 focusing during the filament heating, or peak tailing corrections [6]. Hence, the classical 157 method is more adapted for minor isotope ratios determination $\left(\right.$ e.g. ${ }^{234} \mathrm{U} /{ }^{238} \mathrm{U}$ and $158{ }^{236} \mathrm{U} /{ }^{238} \mathrm{U}$ isotope ratios) because it allows the application of corrections through different 159 measurement sequences [2]. On the other hand, the classical method is affected by 160 isotope fractionation, as it is its main cause of the measurement bias, and requires another 161 mathematical correction. In the present study, when applying the classical method, the 162 isotope fractionation was corrected using an internal normalization established from the 163 major uranium isotope ratio. This isotope ratio (e.g. ${ }^{235} \mathrm{U} /{ }^{238} \mathrm{U}$ isotope ratio) was 164 previously determined using the TE method for major isotope ratio determination.

165 A classical multi-dynamic method (hereafter referred to as CMD method) was developed 166 to measure the ${ }^{234} \mathrm{U} /{ }^{238} \mathrm{U}$ and ${ }^{236} \mathrm{U} /{ }^{238} \mathrm{U}$ isotope ratios. This method includes 4 167 measurement sequences performed one after the other in order to apply several 168 corrections and collect all of the uranium isotopes (Table 1).

169 In the first sequence, the magnetic field was set to collect the ${ }^{236} \mathrm{U}$ isotope on the SEM. 170 The other detectors (Faraday cups) were positioned to collect all of the other uranium 171 isotopes: the ${ }^{234} \mathrm{U}$ isotope was collected on the FC 12 (L2 cup), the ${ }^{235} \mathrm{U}$ (L1 cup) and $172{ }^{238} \mathrm{U}$ (H2 cup) isotopes were collected on a FC 11. An idle time of $1 \mathrm{~s}$ was applied and the 173 measurement was performed with 5 integrations of $4 \mathrm{~s}$. The idle time is necessary to 
174 avoid any drift due to the different time response of the different detectors (the SEM is

175 faster than the FC 11 and the FC 11 is faster than the FC 12).

176 In the second sequence, the magnetic field was modified to collect the ${ }^{234} \mathrm{U}$ isotope on the

177 SEM. The H1 cup, not used in sequence 1, was positioned to collect the ${ }^{235} \mathrm{U}$ on a FC 11.

178 This step was dedicated to the real-time SEM/FC inter-calibration. It was calculated 179 using the ${ }^{234} \mathrm{U} /{ }^{235} \mathrm{U}$ isotope ratio in order to avoid any signal fluctuation and decrease the 180 uncertainty. The inter-calibration was performed by comparing the ${ }^{234} \mathrm{U} /{ }^{235} \mathrm{U}$ isotope ratio 181 measured in sequence 1 using FC 12 and FC 11 with the ${ }^{234} \mathrm{U} /{ }^{235} \mathrm{U}$ isotope ratio measured 182 in sequence 2 using SEM and FC 11. An idle time of $1 \mathrm{~s}$ was applied. The measurement 183 was performed with 5 integrations of $4 \mathrm{~s}$ in order to obtain a good estimation of the 184 SEM/FC inter-calibration.

185 In the third sequence, the magnetic field of the sector field was modified so that the SEM 186 was set at 235.7 amu (i.e. ${ }^{236} \mathrm{U}-0.35,{ }^{236} \mathrm{U}$ mass being about $236.05 \mathrm{amu}$ ) for tailing 187 contribution measurement. It was measured closer to the peak in order to make the linear 188 interpolation more accurate. This step measured in parallel the tailing contribution at 189 mass ${ }^{234} \mathrm{U}-0.35$. The Faraday cups used in this sequence were the same as in sequence 190 1. The signal intensities measured during sequence 3 were weak compared to the signal 191 intensities in sequence 1. An idle time is necessary to ensure that the Faraday cups 192 response return to their background level before the measurement in sequence 3, 193 especially for the FC 12 which has the longest response time. Then, Faraday cups used in 194 sequence 3 had an inaction time of $26 \mathrm{~s}$ before restarting measurement. This time 195 corresponds to the idle time of the sequence $3(5 \mathrm{~s})$ and the sequence 2 total measurement 196 time when the Faraday cups used in sequences 1 and 3 were not collecting any signal (we 197 recall that the idle time of sequence 2 is $1 \mathrm{~s}$ and measurement time of sequence 2 is $1984 \times 5$ s).

199 In the fourth sequence, the magnetic field was modified to collect at the central detector 200 mass 236.4 , corresponding to ${ }^{236} U+0.35(\approx 236.05+0.35)$. This step measured the 201 tailing contribution at mass ${ }^{234} U+0.35$ and ${ }^{236} U+0.35$. The measurement of sequence 3 
202 and 4 was performed with 2 integrations of $4 \mathrm{~s}$, which is sufficient to have a good 203 estimation of the peak tailing contribution.

204 While using the CMD method, the uranium deposit quantity was about $4 \mu \mathrm{g}$. After 205 introducing the filaments inside the TIMS source, the beginning of the method was 206 identical to the TE method: ionization and evaporation filament heating, peak centering, 207 ion focusing and electronic baselines measurements prior to data acquisition. Contrary to 208 the TE method, where the sequence started straight after the electronic baselines, the 209 CMD sequences started when the desired intensity for the ${ }^{234} \mathrm{U}^{+}$ion beam (between 10 210 and $50 \mathrm{mV}$ on the FC 12 in sequence 1) was obtained. These intensities were chosen in 211 order to perform the SEM/FC inter-calibration with a good accuracy: a minimum ${ }^{234} \mathrm{U}^{+}$ 212 ion beam intensity of $1 \times 10^{-14} \mathrm{~A}$ was reached, corresponding to a significant signal of $21310 \mathrm{mV}$ or higher on the FC 12 (sequence 1). This signal also corresponded to about $21462500 \mathrm{cps}$ on the SEM (sequence 2), which is low enough for the ${ }^{234} \mathrm{U}$ isotope intensity 215 to not saturate the detector and reduce significantly its lifespan (the recommended signal 216 in the SEM is < $1000000 \mathrm{cps}$ ). The evaporation filament temperature was controlled to 217 keep the ion beam intensity constant during the measurement, by increasing the 218 evaporation current when necessary. Each measurement corresponded to 6 blocks of 10 219 cycles. Each cycle corresponded to the acquisition of the 4 measurement sequences 220 presented in the Table 1. The baseline, the "peak center" and the lens optimization were 221 performed every 2 blocks. After each block, the amplifiers $10^{11} \Omega$ connected to the 222 Faraday cups rotated for permitting each Faraday cups to connect to each used amplifier 223 during the analysis.

224 Among the different fractionation laws (linear law, power law, exponential law or 225 Rayleigh law), the exponential law was found to be the best approach for many elements $226[10,13,14]$. It was then used for the isotope fractionation correction (Eq. (1)).

$$
R_{\text {corr }}=R_{\text {meas }} \times\left(\frac{M_{i}}{M_{j}}\right)^{p}
$$


227 Where $p$ is the normalization factor. $R_{\text {corr }}$ is the ${ }^{234} U /{ }^{238} U$ or ${ }^{236} U{ }^{238} U$ corrected isotope 228 ratio. $R_{\text {meas }}$ is the ${ }^{234} U /{ }^{238} U$ or ${ }^{236} U /{ }^{238} U$ measured isotope ratio in the sequence 1 and $M_{i}$ 229 and $\mathrm{M}_{\mathrm{j}}$ are the molar masses of the isotope involved in the isotope ratio.

230 The normalization factor was obtained using Eq. (2):

$$
p=\frac{\ln \left(\frac{\left(\frac{{ }^{235} U}{238 U}\right)_{\text {cert }}}{\left(\frac{235 U}{238 U}\right)_{\text {meas }}}\right)}{\ln \left(\frac{M_{235}}{M_{238_{U}}}\right)}
$$

231 Where $\left({ }^{235} \mathrm{U} /{ }^{238} \mathrm{U}\right)$ cert is the ${ }^{235} \mathrm{U} /{ }^{238} \mathrm{U}$ certified isotope ratio for the U015 CRM or the ratio 232 measured using the TE method for the RRT sample. $\left({ }^{235} \mathrm{U} /{ }^{238} \mathrm{U}\right)_{\text {meas }}$ is the ${ }^{235} \mathrm{U} /{ }^{238} \mathrm{U}$ 233 isotope ratio measured in sequence $1 . \mathrm{M}_{235_{\mathrm{U}}}$ and $\mathrm{M}_{238_{\mathrm{U}}}$ are the molar masses of the ${ }^{235} \mathrm{U}$ 234 and ${ }^{238} \mathrm{U}$ isotopes.

235 The ${ }^{234} \mathrm{U} /{ }^{238} \mathrm{U}$ isotope ratio $\left((234 / 238)_{\text {corr }}\right)$, corrected from the peak tailing and the isotope 236 fractionation, is then given by Eq. (3):

$$
\left(\frac{234}{238}\right)_{c o r r}=\left(\left(\frac{234}{238}\right)_{\text {meas }}-\frac{1}{2}\left(\frac{233.7}{238}+\frac{234.4}{238}\right)\right)\left(\frac{M_{234} U}{M_{238} U}\right)^{p}
$$

237 The $(234 / 238)_{\text {meas }}$ isotope ratio is the ${ }^{234} \mathrm{U} /{ }^{238} \mathrm{U}$ isotope ratio measured in sequence 1 . The 238 233.7/238 ratio corresponds to the signal measured on the L2 Faraday cup in sequence 3 239 over the signal of ${ }^{238} \mathrm{U}$ in sequence 1 . The $234.4 / 238$ ratio corresponds to the signal 240 measured on the L2 Faraday cup in sequence 4 over the signal of ${ }^{238} \mathrm{U}$ in sequence 1. $241 \mathrm{M}_{234 \mathrm{U}}$ and $\mathrm{M}_{238_{\mathrm{U}}}$ are the molar masses of the ${ }^{234} \mathrm{U}$ and ${ }^{238} \mathrm{U}$ isotopes. $\mathrm{p}$ is the 242 normalization factor obtained from Eq. (2).

243 The ${ }^{236} \mathrm{U}^{238} \mathrm{U}$ isotope ratio $\left((236 / 238)_{\text {corr }}\right)$ corrected from the peak tailing, the SEM/FC 244 inter-calibration gain and the isotope fractionation is given by Eq. (4).

$$
\left(\frac{236}{238}\right)_{c o r r}=\frac{1}{G}\left(\left(\frac{236}{238}\right)_{\text {meas }}-\frac{1}{2}\left(\frac{235.7}{238}+\frac{236.4}{238}\right)\right)\left(\frac{M_{236} U}{M_{238}}\right)^{p}
$$


245 Where $\mathrm{G}$ is the SEM/FC inter-calibration gain. The $(236 / 238)_{\text {meas }}$ isotope ratio is the $246{ }^{236} \mathrm{U}^{238} \mathrm{U}$ isotope ratio measured in sequence 1. The 235.7/238 ratio corresponds to the 247 signal measured on the SEM in sequence 3 over the signal of ${ }^{238} U$ in sequence 1 . The 248 236.4/238 ratio corresponds to the signal measured on the SEM in sequence 4 over the 249 signal of ${ }^{238} \mathrm{U}$ in sequence $1 . \mathrm{M}_{236 \mathrm{U}}$ and $\mathrm{M}_{238_{\mathrm{U}}}$ are the molar masses of the ${ }^{234} \mathrm{U}$ and ${ }^{238} \mathrm{U}$ 250 isotopes. $p$ is the normalization factor obtained from Eq. (2).

251 The SEM/FC inter-calibration gain was calculated using Eq. (5). It includes the peak 252 tailing correction from the ${ }^{235} \mathrm{U}^{+}$and ${ }^{238} \mathrm{U}^{+}$ion beams to the ${ }^{234} \mathrm{U}^{+}$beam detection on the 253 FC 12. No peak tailing correction was applied to the SEM since the RPQ energy filter 254 helps decreasing the peak tailing by 2 orders of magnitude $[4,10]$.

$$
G=\left(\frac{\left(\frac{234}{235}\right)_{S 2}}{\left(\frac{234}{235}\right)_{S 1}}\right) \times \frac{\left(\frac{234}{238}\right)_{S 1}}{\left(\frac{234}{238}\right)_{S 1}-\frac{1}{2}\left(\frac{233.7}{238}+\frac{234.4}{238}\right)}
$$

255 Where S1 and S2 are isotope ratio measured in sequence 1 or 2, respectively. The $256233.7 / 238$ ratio corresponds to the signal measured on the L2 Faraday cup in sequence 3 257 over the signal of ${ }^{238} \mathrm{U}$ in sequence 1 . The $234.4 / 238$ ratio corresponds to the signal 258 measured on the L2 Faraday cup in sequence 4 over the signal of ${ }^{238} \mathrm{U}$ in sequence 1 .

259 The ${ }^{234} \mathrm{U} /{ }^{238} \mathrm{U}$ and ${ }^{236} \mathrm{U} /{ }^{238} \mathrm{U}$ corrected isotope ratios were calculated during each 260 measurement cycle. After the end of the measurement (i.e. after the 6 blocks of 10 cycles) 261 a statistical test rejecting the values outside the average plus or minus twice the standard 262 deviation was applied twice for both isotope ratios. Around $7 \%$ of the values were 263 rejected. The ${ }^{234} \mathrm{U} /{ }^{238} \mathrm{U}$ and ${ }^{236} \mathrm{U} /{ }^{238} \mathrm{U}$ corrected isotope ratios were obtained by the 264 average of the non-rejected values.

265 For the method validation, 4 analyses were performed on the U015 CRM. For the RRT 266 sample determination, 5 analyses were performed. 
270 Bias, or trueness, was calculated using Eq. (6).

$$
\operatorname{Bias}(\%)=\frac{Z-\operatorname{cert}}{\operatorname{cert}} \times 100
$$

271 Where $\mathrm{Z}$ is the experimental value and cert is the reference value of the CRM used to 272 evaluate the method trueness or the RRT assigned value.

273 According to the NF T 90-210 norm, Eq. (7) was used to determine if the analytical 274 method has a statistically significant bias [15]. If the normalized bias (NB) is lower than 2752 , the method is considered having no statistically significant bias.

$$
N B=\frac{\mid Z-\text { cert } \mid}{\sqrt{s^{2}+u_{\text {cert }}^{2}}}
$$

276 Where $s$ is the standard deviation of the different measurements and $\mathrm{u}_{\text {cert }}$ is the CRM or 277 assigned value uncertainty with a coverage factor at $\mathrm{k}=1$.

278 The precision of the different methods was evaluated by calculating the Relative Standard 279 Deviation (RSD) of all the measurements.

280 The isotope ratio measurement uncertainties estimation was described in previous work 281 [12]. The isotope ratio (R) uncertainty (u) at $\mathrm{k}=1$ was estimated using Eq. (8).

$$
\begin{aligned}
& \frac{u^{2}(\mathrm{R})}{(\mathrm{R})^{2}}=\frac{u^{2}(\bar{x})}{\bar{x}^{2}}+\frac{u^{2}(\text { trueness })}{(\text { trueness })^{2}}+\frac{u^{2}(\text { cert })}{(\text { cert })^{2}} \\
& \frac{u(\text { trueness })}{\text { trueness }}=\frac{\text { Maximum bias on } C R M}{\sqrt{3}}
\end{aligned}
$$


282

283

284

285

286

287

288

289

290

291

292

293

294

295

296

297

298

299

300

301

302

303

304

305

306

307

The first term of Eq. (8) includes the uncertainty from the random effects and is given by the RSD of all the measurements (i.e. the precision). The second and third terms take into account the systematic effects (i.e. the measurement trueness of the method). The measurement trueness is calculated using Eq. (9) and is determined with the U015 CRM because of its isotopic properties close to the RRT sample.

\section{Results and discussion}

\section{Comparison of isotope measurement methods for minor isotope ratio determination}

The CMD method was compared to the TE method using the FC 12 ("TE FC 12") as well as the TE method using the SEM ("TE SEM") for the determination of the ${ }^{234} \mathrm{U} / 238 \mathrm{U}$ and ${ }^{236} \mathrm{U} /{ }^{238} \mathrm{U}$ minor isotope ratios of the U015 CRM. The results are presented in Fig. 1.

\section{TE method using FC 12}

Using the TE method, the signals measured on the FC 12 were $13 \mathrm{mV}$ for ${ }^{234} \mathrm{U}^{+}$and $25 \mathrm{mV}$ for ${ }^{236} \mathrm{U}^{+}$. These intensities are very weak in comparison to the signal measured for ${ }^{238} \mathrm{U}^{+}(15 \mathrm{~V})$. The theoretical Faraday cup detection limit can be estimated as 3 times the quadratic sum of the standard deviation of the Johnson Nyquist noise and the Poissonnoise (about $0.4 \mathrm{mV}$ for a FC 12) [16,17]. The minor isotope signals are about 30 times (for the ${ }^{234} \mathrm{U}^{+}$) and 60 times (for the ${ }^{236} \mathrm{U}^{+}$) higher than the estimated detection limit of the FC 12. The TE FC 12 method showed a bias of $2.08 \%$ and $4.11 \%$ for the ${ }^{234} \mathrm{U} /{ }^{238} \mathrm{U}$ and ${ }^{236} \mathrm{U} /{ }^{238} \mathrm{U}$ ratios, respectively. Despite the higher ${ }^{236} \mathrm{U}$ intensity $(25 \mathrm{mV})$ compared to the ${ }^{234} \mathrm{U}$ intensity $(13 \mathrm{mV})$, the bias for the ${ }^{236} \mathrm{U} /{ }^{238} \mathrm{U}(4.1 \%)$ is significantly higher than the ${ }^{234} \mathrm{U} /{ }^{238} \mathrm{U}(2.1 \%)$. The low signal intensity is therefore not the only reason for a higher bias. The presence of the ${ }^{238} U$ major isotope, closer to the ${ }^{236} U$ than to the ${ }^{234} U$ isotope, explains the bias difference because of peak tailing issues. The RSD for the ${ }^{234} U /{ }^{238} U$ and ${ }^{236} \mathrm{U} /{ }^{238} \mathrm{U}$ isotope ratios were similar and about $1 \%$. Unlike the normalized bias observed for the ${ }^{234} \mathrm{U} /{ }^{238} \mathrm{U}$ isotope ratio (1.8), the normalized bias for the ${ }^{236} \mathrm{U} /{ }^{238} \mathrm{U}$ isotope ratio 
308 was equal to 3.3 showing that the method has a significant bias for the ${ }^{236} \mathrm{U} /{ }^{238} \mathrm{U}$ ratio.

309 The uncertainties were estimated to $4.3 \%$ for the ${ }^{234} \mathrm{U} /{ }^{238} \mathrm{U}$ ratio and $6.8 \%$ for the

$310{ }^{236} \mathrm{U} /{ }^{238} \mathrm{U}$ ratio, with a major contribution from the systematic error (Table 2).

\section{$311 \quad$ TE method using SEM}

312 The SEM improves the sensitivity in comparison to the FC 12: the measured signals were

$31378000 \mathrm{cps}$ for the ${ }^{234} \mathrm{U}$ isotope and $150000 \mathrm{cps}$ for the ${ }^{236} \mathrm{U}$ isotope. These intensities are

314 much higher than the SEM dark noise (below 10 counts per minute). In comparison to the

315 TE FC 12 method, the bias greatly decreased $\left(0.40 \%\right.$ for the ${ }^{234} \mathrm{U} /{ }^{238} \mathrm{U}$ ratio and $0.18 \%$

316 for the ${ }^{236} \mathrm{U} /{ }^{238} \mathrm{U}$ ratio). This improvement can be due to the better detector sensitivity and

317 to the fact that the SEM is equipped with a RPQ filter that provides a bias reduction for

318 the minor isotope ratio determination. In order to find the best contributor to the

319 improvement of the measurement trueness, 3 additional analyses under the same

320 analytical conditions except that the RPQ filter was not used were performed using the

321 TE method with the SEM to collect the ${ }^{236} \mathrm{U}$ (Fig. 1.b). The measurement trueness was

322 degraded without the RPQ filter: the bias was higher than $5 \%$, while a bias below $0.2 \%$

323 is obtained with the RPQ filter. The normalized bias computed without RPQ filter shows

324 the method has a significant bias $(\mathrm{NB}>2)$. The ${ }^{236} \mathrm{U} /{ }^{238} \mathrm{U}$ ratios determined using the TE

325 FC 12 method and the TE SEM method without RPQ filter were all higher than the

326 certified values (Fig. 1). This is obviously due to the peak tailing effect of ${ }^{238} \mathrm{U}$ as the

327 abundance sensitivity is about $10^{-6}$ without RPQ filter for the Triton TIMS. These

328 observations show when reducing the peak tailing contribution using the RPQ energy

329 filter is the main cause of the measurement trueness improvement. On the other hand, it

330 can be noticed that the use of the RPQ filter reduces the ions intensity by about $5 \%$. This

331 loss is negligible compared to the benefit obtained on the measurement trueness.

332 However, the observed RSD for the ${ }^{234} \mathrm{U} /{ }^{238} \mathrm{U}$ and ${ }^{236} \mathrm{U} /{ }^{238} \mathrm{U}$ obtained with the TE method

333 using the SEM and the RPQ filter (Fig. 1) are high (1.1\% for the ${ }^{234} \mathrm{U} /{ }^{238} \mathrm{U}$ ratio and

$3342.1 \%$ for the ${ }^{236} \mathrm{U} /{ }^{238} \mathrm{U}$ ratio). The poor repeatability is explained by the SEM instability

335 during the measurement. The SEM calibration can vary significantly during an analysis 
336 without any predictable trend [1]. Despite the SEM instability, the estimated uncertainty 337 (2.8\% for the ${ }^{234} \mathrm{U} /{ }^{238} \mathrm{U}$ ratio and $5.0 \%$ for the ${ }^{236} \mathrm{U} /{ }^{238} \mathrm{U}$ ratio) slightly decreased 338 compared to the TE FC 12 method ( $4.3 \%$ for the ${ }^{234} \mathrm{U} /{ }^{238} \mathrm{U}$ ratio and $6.8 \%$ for the $339{ }^{236} \mathrm{U} /{ }^{238} \mathrm{U}$ ratio). The relative contribution of the main uncertainty sources (Table 3) 340 shows that the total uncertainties are mainly due to the precision (55\% for the ${ }^{234} \mathrm{U}{ }^{238} \mathrm{U}$ 341 ratio and $71 \%$ for the ${ }^{236} \mathrm{U} /{ }^{238} \mathrm{U}$ ratio). The method shows no significant bias for both 342 isotope ratios: the normalized biases are below 2 .

344 The biases obtained using the CMD method are equal to $-0.28 \%$ for the ${ }^{234} \mathrm{U} /{ }^{238} \mathrm{U}$ ratio 345 and $0.19 \%$ for the ${ }^{236} \mathrm{U} /{ }^{238} \mathrm{U}$ ratio (Fig. 1 and Table 3). The observed RSD on the ${ }^{234} \mathrm{U} /{ }^{238} \mathrm{U}$ and ${ }^{236} \mathrm{U} /{ }^{238} \mathrm{U}$ are equal to $0.05 \%$ and $0.08 \%$, respectively. The normalized

347 bias calculated for both isotope ratios were below 2, showing the method has no

348 significant bias. The CMD method improves the measurement trueness by decreasing the 349 abundance sensitivity influence using the RPQ filter combined with a mathematical 350 correction. The CMD method also improves the precision due to a "real time" SEM 351 calibration. The impact of the SEM fluctuation is then minimized. The method's internal 352 normalization helps maintaining a good measurement accuracy and correct the isotope 353 fractionation. Different parameters influencing the isotope fractionation, such as the 354 deposit quality, are compensated by the internal normalization. The uncertainties were 355 estimated to $1.2 \%$ for the ${ }^{234} \mathrm{U} /{ }^{238} \mathrm{U}$ ratio and $0.72 \%$ for the ${ }^{236} \mathrm{U} /{ }^{238} \mathrm{U}$ ratio. The main 356 sources of uncertainty for ${ }^{234} U /{ }^{238} U$ and ${ }^{236} U{ }^{238} U$ isotope ratios confirm the significant 357 improvement of the precision and the measurement trueness when applying the CMD 358 method (Table 2): the measurement trueness (11\% for the ${ }^{234} \mathrm{U} /{ }^{238} \mathrm{U}$ ratio and $23 \%$ for 359 the ${ }^{236} U /{ }^{238} U$ ratio) and precision ( $1 \%$ for the ${ }^{234} U^{238} U$ ratio and $5 \%$ for the ${ }^{236} U /{ }^{238} U$ 360 ratio) are minor contributors to the total uncertainty. The main contribution is the CRM 361 uncertainty. The lowest uncertainties are obtained with the CMD method: $362 U(\mathrm{k}=2)=4.3 \%, 2.8 \%$ and $1.2 \%$ for the ${ }^{234} \mathrm{U} /{ }^{238} \mathrm{U}$ isotope ratio using TE FC 12, TE 363 SEM and CMD methods, respectively, and $U(\mathrm{k}=2)=6.8 \%, 5.0 \%$ and $0.72 \%$ for the $364{ }^{236} \mathrm{U} /{ }^{238} \mathrm{U}$ isotope ratio using TE FC 12 , TE SEM and CMD methods, respectively. 
366 These 3 methods have different assets in terms of analysis time, simplicity, trueness and 367 repeatability. The TE FC 12 method is the simplest and the most straightforward method: 368 no inter-calibration gain is required between two sample analyses as the Faraday cups are 369 very stable and all the isotope ratios of an element (major and minor isotope) are directly 370 obtained. However, this method needs to take into account the method bias in the 371 uncertainty calculation. The application of this method is easily transposable to another 372 element assuming that the number of minor isotopes do not exceed the number of FC 12. 373 An analysis using the FC 12 takes generally between 20 to 60 minutes.

374 The TE SEM method is rather simple also. The sample analysis itself has the same 375 duration as the TE method with FC 12 (between 20 to 60 minutes). However, the $376 \mathrm{SEM} / \mathrm{FC}$ inter-calibration gain is required before and after each sample analysis. This 377 explains the longer total analysis time (each inter-calibration gain takes about 20 378 minutes). The method gives directly all the isotope ratios of an element (major and minor 379 isotope). Depending on the number of SEM detectors available in the instrument, the 380 analysis might require several runs. Also, this method can be easily transposable to 381 another element.

382 The CMD method is the most complex one. However, once the file for the ${ }^{234} \mathrm{U} /{ }^{238} \mathrm{U}$ and $383{ }^{236} \mathrm{U} /{ }^{238} \mathrm{U}$ ratios computation is created, the method can be easily used put in routine 384 analysis. The method requires bigger sample amount (about $4 \mu \mathrm{g}$ ) than the TE method 385 (less than $1 \mu \mathrm{g}$ ) in order to keep a high signal during the entire analysis that lasts about 90 386 minutes. Also, this method requires the results of others analyses: the ${ }^{235} \mathrm{U} /{ }^{238} \mathrm{U}$ isotope 387 ratio determination with a high accuracy method, like the TE method, is needed to 388 perform the isotope fractionation correction of the ${ }^{234} U /{ }^{238} U$ and ${ }^{236} U /{ }^{238} U$ ratios, which 389 increases the overall analysis time. This method is dedicated to the situation where high 390 accuracy minor isotope ratio determination is necessary. The method can be transposable 391 to enriched uranium. However, for depleted uranium, the ${ }^{234} \mathrm{U} /{ }^{238} \mathrm{U}$ isotope ratio is 392 generally too low to perform both the measurement of the ${ }^{234} \mathrm{U} /{ }^{238} \mathrm{U}$ isotope ratio and the 393 SEM/FC inter-calibration in the same method. Indeed, the SEM/FC inter-calibration 
394 requires a minimum signal of $10 \mathrm{mV}$ with the FC 12. Considering that for depleted 395 uranium, the ${ }^{234} \mathrm{U} /{ }^{238} \mathrm{U}$ isotope ratios are below $2 \times 10^{-5}$, the signal with FC 11 for the ${ }^{238} \mathrm{U}$ 396 isotope measurement would exceed its maximum limit (50 V). So, for depleted uranium 397 using the CMD method, the ${ }^{234} \mathrm{U} /{ }^{238} \mathrm{U}$ and ${ }^{236} \mathrm{U} /{ }^{238} \mathrm{U}$ isotope ratios are not directly 398 accessible. These minor ratios could be obtained indirectly using the measurements of the

$399{ }^{234} \mathrm{U} /{ }^{235} \mathrm{U}$ and ${ }^{236} \mathrm{U} /{ }^{235} \mathrm{U}$ isotope ratios and by knowing the ${ }^{235} \mathrm{U} /{ }^{238} \mathrm{U}$ isotope ratio. In this 400 case, the CMD method would need adjustment: the internal normalization would be 401 performed with the measurement of the ${ }^{234} \mathrm{U} /{ }^{235} \mathrm{U}$ as the ${ }^{238} \mathrm{U}$ isotope would not be 402 collected. In a more general point of view, the CMD method needs adjustments for each 403 element to be measured. The analyzed element needs to have a minimum of 3 isotopes: 404 one major isotope, one minor isotope needing a measurement with the SEM and an 405 "intermediate" isotope allowing the in situ SEM/FC inter-calibration. This last isotope 406 needs a significantly lower abundance compared to the major isotope and a significantly 407 higher abundance compared to the minor isotope.

408 The CMD method shows also some similarities with the measurement sequence of the 409 MTE method [10]. The main difference between the MTE and the CMD methods comes 410 from the isotope fractionation correction: total evaporation for the MTE method or 411 internal normalization for the CMD method. The MTE method is the reference method in 412 order to have the lowest uncertainties. However, the CMD method presents some 413 advantages compared to the MTE method. The principal advantages is a shorter analysis 414 time: the CMD method take about 90 min compared to the 3-5 hours for the MTE 415 method. The CMD method is also simpler to configure in the TIMS software. The CMD 416 method is directly configurable in the TIMS software without the requirement of an 417 external script [10].

$418 \quad$ Method validation

419 The results for the method validation on the U015 CRM are summarized in Table 2. The 420 CMD method shows lower bias, better repeatability and uncertainty compared to the TE 421 method for the ${ }^{234} \mathrm{U} /{ }^{238} \mathrm{U}$ and ${ }^{236} \mathrm{U} /{ }^{238} \mathrm{U}$ isotope ratios measurements. The CMD method 
422 displayed no significant bias (normalized bias below to 2). These results validate the $423{ }^{234} \mathrm{U} /{ }^{238} \mathrm{U}$ and ${ }^{236} \mathrm{U} /{ }^{238} \mathrm{U}$ isotope ratios determination using the CMD method.

424 The result obtained for the ${ }^{235} \mathrm{U} /{ }^{238} \mathrm{U}$ is in good agreement with the certified value. The 425 bias obtained is equal to $0.03 \%$ and the RSD is $0.02 \%$. The normalized bias is equal to 4260.62 , showing that the method leads to no significant bias. The uncertainty is estimated to $4270.16 \%(\mathrm{k}=2)$ and is in compliance with the safeguard requirements given by the ITV $428(0.28 \%, \mathrm{k}=2)$ on this range of uranium isotopic composition [18]. It should be noticed 429 that the first five experiments were performed before the RRT sample isotope 430 measurement and the last one after the RRT sample isotope measurement (see next 431 section), ensuring that the instrumental performance is satisfactory during the whole 432 measurement series. These results validate the ${ }^{235} \mathrm{U} /{ }^{238} \mathrm{U}$ isotope ratio determination using 433 the TE method.

435 The RRT sample was analyzed as an unknown sample. However, at the end of the study, 436 the results were compared to the RRT assigned values in order to evaluate the developed 437 methodology.

438 The method developed and validated using the U015 CRM was applied. The ${ }^{235} \mathrm{U} /{ }^{238} \mathrm{U}$ 439 ratio measurements with the FC 11 or the FC 12 for the ${ }^{235} \mathrm{U}$ detection show no 440 significant bias: biases are below $0.1 \%$ and the normalized biases are below 2 (Table 4). 441 The measurements seem to demonstrate that the precision is slightly better using the FC $44212(\mathrm{RSD}=0.03 \%)$ than using the $\mathrm{FC} 11(\mathrm{RSD}=0.06 \%)$.

443 The results obtained for the ${ }^{234} \mathrm{U} /{ }^{238} \mathrm{U}$ (bias $=0.27 \%$ ), ${ }^{235} \mathrm{U} /{ }^{238} \mathrm{U}$ (bias $=0.07 \%$ ) and $444{ }^{236} \mathrm{U} /{ }^{238} \mathrm{U}$ (bias $=0.93 \%$ ) isotope ratios are in good agreement with the assigned values 445 provided by IAEA (Table 4). The RSD are similar to the ones obtained for the U015 $446 \mathrm{CRM}$ and are lower than $0.1 \%: 0.07 \%, 0.04 \%$ and $0.08 \%$ for the ${ }^{234} \mathrm{U} /{ }^{238} \mathrm{U},{ }^{235} \mathrm{U} /{ }^{238} \mathrm{U}$ 447 and ${ }^{236} \mathrm{U} /{ }^{238} \mathrm{U}$ isotope ratios, respectively. The ${ }^{235} \mathrm{U} /{ }^{238} \mathrm{U}$ isotope ratio uncertainty is 448 estimated at $0.15 \%(\mathrm{k}=2)$ and is in compliance with the ITV $(0.28 \%, \mathrm{k}=2)$ for this 
449 type of enriched uranium [18]. The relative contribution, expressed in percent, of the 450 main uncertainty sources for the uranium isotope ratios determination is given in Table 2.

451 The three uncertainty sources considered for the ${ }^{235} \mathrm{U} /{ }^{238} \mathrm{U}$ isotope ratio determination 452 have a similar contribution: U015 CRM certified isotope ratio (41\%), precision (31\%) 453 and method trueness $(28 \%)$. The ${ }^{234} \mathrm{U} /{ }^{238} \mathrm{U}$ isotope ratio uncertainty is estimated at $4541.13 \%(\mathrm{k}=2)$. The uncertainty associated with the CRM isotope ratio is the main 455 uncertainty source in the final uncertainty (87\%). The uncertainties associated with the 456 method bias (11\%) and the precision (2\%) have a limited impact on the final 457 uncertainty. The ${ }^{236} \mathrm{U} /{ }^{238} \mathrm{U}$ isotope ratio uncertainty is estimated at $0.72 \%(\mathrm{k}=2)$. In the 458 same way as the ${ }^{234} \mathrm{U} /{ }^{238} \mathrm{U}$, the final uncertainty mostly comes from the CRM isotope 459 ratio uncertainty $(72 \%)$.

460 The methodology developed to perform uranium isotope ratio with high accuracy showed 461 no significant bias: all the normalized biases are lower than 2.

\section{Conclusions}

463 This study shows the possibility of the TE and the CMD methods for determining 464 uranium isotope ratios with low uncertainties. The TE method allows to reach the ITV 465 requirements for the ${ }^{235} \mathrm{U} /{ }^{238} \mathrm{U}$ major isotope ratio. One of the methods for the ${ }^{234} \mathrm{U} /{ }^{238} \mathrm{U}$ 466 and ${ }^{236} \mathrm{U} /{ }^{238} \mathrm{U}$ minor isotope ratios determination was the classical method using multi467 dynamic sequences. This classical method allows mathematical correction of the 468 abundance sensitivity and calibrating the SEM detector while the method is running. An 469 internal normalization using the ${ }^{235} \mathrm{U} /{ }^{238} \mathrm{U}$ major isotope ratio was used to overcome the 470 isotope fractionation. The CMD method decreases the bias, the repeatability and the 471 estimated uncertainties compared to the TE method for uranium minor isotope ratios 472 determination, which is also an interesting feature for forensic analysis applications.

473 Another application of the accurate determination of the pellet isotope composition is the 474 uranium content determination with high accuracy using isotope dilution, a method that 475 will be presented in future. 
477 We are grateful to Dr. S. Baghdadi (IRSN/PSN-EXP/SSRD/BTE) for her precious advice 478 on the present paper. We would like to thank the entire IAEA staff who organized the 479 round robin test (Seibersdorf-Austria). 
Table 1: Summarized description of one cycle of the CMD method

\begin{tabular}{ccccccccc}
\hline Cups & L2 & L1 & C & H1 & H2 & $\begin{array}{c}\text { Number of } \\
\text { integrations }\end{array}$ & $\begin{array}{c}\text { Measurement } \\
\text { time (s) }\end{array}$ & $\begin{array}{c}\text { Idle time } \\
\text { (s) }\end{array}$ \\
Detectors & FC 12 & FC 11 & SEM & FC 11 & FC 11 & & \\
Sequence 1 & ${ }^{234} \mathrm{U}$ & ${ }^{235} \mathrm{U}$ & ${ }^{236} \mathrm{U}$ & & ${ }^{238} \mathrm{U}$ & 5 & 4 & 1 \\
Sequence 2 & & & ${ }^{234} \mathrm{U}$ & ${ }^{235} \mathrm{U}$ & & 5 & 4 & 1 \\
Sequence 3 & 233.7 & 234.7 & 235.7 & & 237.7 & 2 & 4 & 5 \\
Sequence 4 & 234.4 & 235.4 & 236.4 & & 238.4 & 2 & 4 & 1 \\
\hline
\end{tabular}

482

483

484 Table 2: Relative contribution (\%) of the main uncertainty sources for the ${ }^{234} \mathrm{U}{ }^{238} \mathrm{U}$ and $485{ }^{236} \mathrm{U}^{238} \mathrm{U}$ isotope ratios measurement with the TE method using the FC 12 (TE FC 12) or 486 the SEM (TE SEM) and with the CMD method (CMD), and for the ${ }^{235} \mathrm{U} /{ }^{238} \mathrm{U}$ isotope $487 \quad$ ratio with the TE method (TE) for the U015 CRM and the RRT sample (RRT)

\begin{tabular}{cccccc}
\hline $\begin{array}{c}\text { Isotope } \\
\text { ratio }\end{array}$ & Sample & Method & \multicolumn{3}{c}{ Relative contribution (\%) } \\
& U015 & TE FC 12 & 21 & 73 & 6 \\
& U015 & TE SEM & 55 & 31 & 14 \\
${ }^{234} \mathrm{U} /{ }^{238} \mathrm{U}$ & $\mathrm{U} 015$ & CMD & 1 & 11 & 88 \\
& RRT & CMD & 2 & 11 & 87 \\
\hline \multirow{2}{*}{${ }^{235} \mathrm{U} /{ }^{238} \mathrm{U}$} & $\mathrm{U} 015$ & TE & 13 & 36 & 51 \\
& RRT & TE & 29 & 29 & 42 \\
\hline \multirow{2}{*}{${ }^{236} \mathrm{U} /{ }^{238} \mathrm{U}$} & $\mathrm{U} 015$ & TE FC 12 & 12 & 87 & 1 \\
& $\mathrm{U} 015$ & TE SEM & 71 & 27 & 2 \\
& $\mathrm{U} 015$ & CMD & 5 & 23 & 72 \\
& RRT & CMD & 5 & 23 & 72 \\
\hline
\end{tabular}

488

489 
491 Table 3: Isotope ratios obtained on the U015 CRM with the TE method (TE) and the 492 CMD method (CMD). Cert. corresponds to the CRM certified value, $U_{\text {cert }}$ corresponds to 493 the CRM certified value uncertainty $(\mathrm{k}=2)$ and $U$ corresponds to the total uncertainty 494 $(\mathrm{k}=2)$. NB corresponds to the normalized bias.

\begin{tabular}{cccc}
\hline $\begin{array}{c}\text { Isotope ratio } \\
\text { Method }\end{array}$ & ${ }^{234} \mathrm{U} /{ }^{238} \mathrm{U}$ & ${ }^{235} \mathrm{U} /{ }^{238} \mathrm{U}$ & ${ }^{236} \mathrm{U} /{ }^{238} \mathrm{U}$ \\
Cert. & 0.00008634 & 0.015565 & 0.0001666 \\
Results & 0.00008610 & 0.015570 & 0.0001669 \\
RSD (\%) & 0.05 & 0.02 & 0.08 \\
Bias (\%) & -0.28 & 0.03 & 0.19 \\
NB & 0.53 & 0.62 & 0.61 \\
$U_{\text {cert }}(\%)$ & 1.07 & 0.10 & 0.60 \\
$U(\%)$ & 1.13 & 0.16 & 0.72 \\
\hline
\end{tabular}

495

496 Table 4: Isotope ratios obtained with the TE method using the FC 11 (TE FC 11) or the 497 FC 12 (TE FC 12) and with the CMD method (CMD) on the RRT uranium pellet. $U_{\text {ass_val. }}$ 498 corresponds to the assigned value uncertainty $(\mathrm{k}=2)$ and $U$ corresponds to the total 499 uncertainty $(\mathrm{k}=2)$. NB corresponds to the normalized bias

\begin{tabular}{ccc||ccc}
\hline Isotope ratio & ${ }^{234} \mathrm{U} /{ }^{238} \mathrm{U}$ & ${ }^{236} \mathrm{U} /{ }^{238} \mathrm{U}$ & \multicolumn{3}{c}{${ }^{235} \mathrm{U} /{ }^{238} \mathrm{U}$} \\
Method & $\mathrm{CMD}$ & $\mathrm{CMD}$ & $\mathrm{TE}$ & $\mathrm{TE}$ & $\begin{array}{c}\text { TE } \\
\text { Average }\end{array}$ \\
\hline Assigned value & 0.0001708 & 0.0000258 & 0.019645 & 0.019645 & 0.019645 \\
Results & 0.0001713 & 0.00002604 & 0.019658 & 0.019659 & 0.019658 \\
RSD (\%) & 0.07 & 0.08 & 0.06 & 0.03 & 0.04 \\
Bias (\%) & 0.27 & 0.93 & 0.06 & 0.07 & 0.07 \\
NB & 0.56 & 0.24 & 0.94 & 1.5 & 1.2 \\
$U_{\text {ass_val. }}(\%)$ & 0.94 & 7.8 & 0.08 & 0.08 & 0.08 \\
$U(\%)$ & 1.13 & 0.72 & 0.18 & 0.15 & 0.15 \\
\hline
\end{tabular}


503

504

505

506

507

508

509

510

511
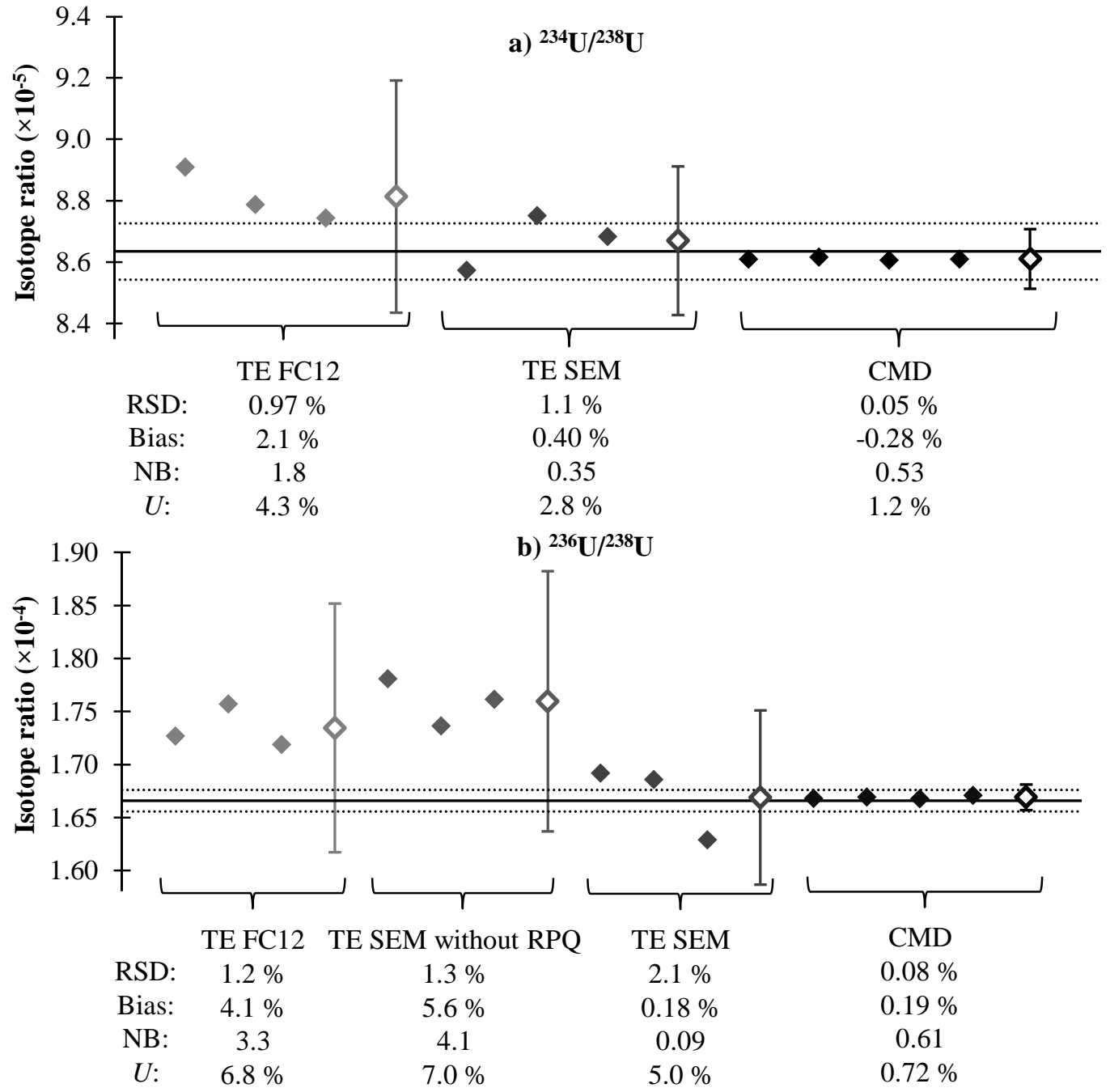

Fig. 1: ${ }^{234} U{ }^{238} \mathrm{U}$ (a) and ${ }^{236} \mathrm{U} /{ }^{238} \mathrm{U}$ (b) isotope ratios measurement on the U015 CRM (full diamonds) with the TE method using the FC 12 (TE FC 12) and the SEM (TE SEM) and with the CMD method (CMD). Empty diamonds with error bars represent the series average with its estimated uncertainties at $\mathrm{k}=2$. $U$ corresponds to the total uncertainty $(\mathrm{k}=2)$. NB corresponds to the normalized bias. The full line (-) corresponds to the certified value and the dotted line (...) represents its uncertainty at $\mathrm{k}=2$ 


\section{References}

513 [1] A. Quemet, M. Maloubier and A. Ruas, Contribution of the Faraday cup coupled to $10^{12}$ ohms current amplifier to uranium $235 / 238$ and $234 / 238$ isotope ratio measurements by Thermal Ionization Mass Spectrometry, Int. J. Mass Spectrom. 404 (2016) 35-39. doi:10.1016/j.ijms.2016.04.005.

517 [2] A. Quemet, M. Maloubier, V. Dalier and A. Ruas, Development of an analysis method of minor uranium isotope ratio measurements using electron multipliers in thermal ionization mass spectrometry, Int. J. Mass Spectrom. 374 (2014) 26-32. doi:10.1016/j.ijms.2014.10.008.

3] M.L.D.P. Godoy, J.M. Godoy, L.A. Roldão and L. Tauhata, Determination of total content and isotopic compositions of plutonium and uranium in environmental samples for safeguards purposes by ICP-QMS, J. Environ. Radioact. 100 (2009) 613-625. doi:10.1016/j.jenvrad.2009.04.012.

4] S. Richter, A. Alonso, W. De Bolle, R. Wellum and P.D.P. Taylor, Isotopic "fingerprints" for natural uranium ore samples, Int. J. Mass Spectrom. 193 (1999) 9-14. doi:10.1016/S1387-3806(99)00102-5.

[5] W. Bu, J. Zheng, M.E. Ketterer, S. Hu, S. Uchida and X. Wang, Development and application of mass spectrometric techniques for the ultra-trace determination of 236 U in environmental samples-A review, Anal. Chim. Acta. 995 (2017) 1-20. doi:10.1016/j.aca.2017.09.029.

[6] S.K. Aggarwal, Thermal ionisation mass spectrometry (TIMS) in nuclear science and technology - a review, Anal. Methods. 8 (2016) 942-957. doi:10.1039/c5ay02816g.

535 [7] K.J. Mathew and A. Hasozbek, Comparison of mass spectrometric methods (TE, 536 MTE and conventional) for uranium isotope ratio measurements, J. Radioanal. Nucl. Chem. 307 (2016) 1681-1687. doi:10.1007/s10967-015-4484-8. 
538 [8] A. Quemet, C. Maillard and A. Ruas, Determination of zirconium isotope composition and concentration for nuclear sample analysis using Thermal Ionization Mass Spectrometry, Int. J. Mass Spectrom. 392 (2015) 34-40. doi:10.1016/j.ijms.2015.08.023.

542 [9] A. Trinquier and P. Komander, Precise and accurate uranium isotope analysis by

[10] S. Richter, H. Kühn, Y. Aregbe, M. Hedberg, J. Horta-Domenech, K. Mayer, E. modified total evaporation using $10^{13} \mathrm{ohm}$ current amplifiers, J. Radioanal. Nucl. Chem. 307 (2016) 1927-1932. doi:10.1007/s10967-015-4400-2.

553

554 Zuleger, S. Bürger, S. Boulyga, A. Köpf, J. Poths and K. Mathew, Improvements in routine uranium isotope ratio measurements using the modified total evaporation method for multi-collector thermal ionization mass spectrometry, J. Anal. At. Spectrom. 26 (2011) 550-564. doi:10.1039/C0JA00173B.

[11] C.A. Degueldre, The Analysis of Nuclear Materials and Their Environments Chapter 3.2.5.1. Photon-Induced Mass Spectrometry, 2017. doi:10.1007/978-3319-58006-7.

[12] A. Quemet, A. Ruas, V. Dalier and C. Rivier, Americium isotope analysis by Thermal Ionization Mass Spectrometry using the Total Evaporation Method, Int. J. Mass Spectrom. 431 (2018) 8-14. doi:10.1016/j.jims.2018.05.017.

[13] W.A. Russell, D.A. Papanastassiou and T.A. Tombrello, Ca isotope fractionation on the Earth and other solar system materials, Geochim. Cosmochim. Acta. 42 (1978) 1075-1090. doi:10.1016/0016-7037(78)90105-9.

[14] M. Garçon, M. Boyet, R.W. Carlson, M.F. Horan, D. Auclair and T.D. Mock, Factors influencing the precision and accuracy of $\mathrm{Nd}$ isotope measurements by thermal ionization mass spectrometry, Chem. Geol. 476 (2017) 493-514. doi:10.1016/j.chemgeo.2017.12.003.

[15] AFNOR, NF T90-210 norm: Water quality - Protocol for the intial method performance assesment in a laboratory, 2009. 
565 [16] D. Wielandt and M. Bizzarro, A TIMS-based method for the high precision measurements of the three-isotope potassium composition of small samples, J. Anal. At. Spectrom. 26 (2011) 366.

568 [17] J.M. Koornneef, C. Bouman, J.B. Schwieters and G.R. Davies, Use of 10(12) ohm current amplifiers in $\mathrm{Sr}$ and $\mathrm{Nd}$ isotope analyses by TIMS for application to subnanogram samples, J. Anal. At. Spectrom. 28 (2013) 749. doi:10.1039/c3ja30326h.

571 [18] International Atomic Energy Agency, International Target Values 2010 for 572 Measurement Uncertainties in Safeguarding Nuclear Materials - STR368, Vienna, $573 \quad$ Austria, 2010. 


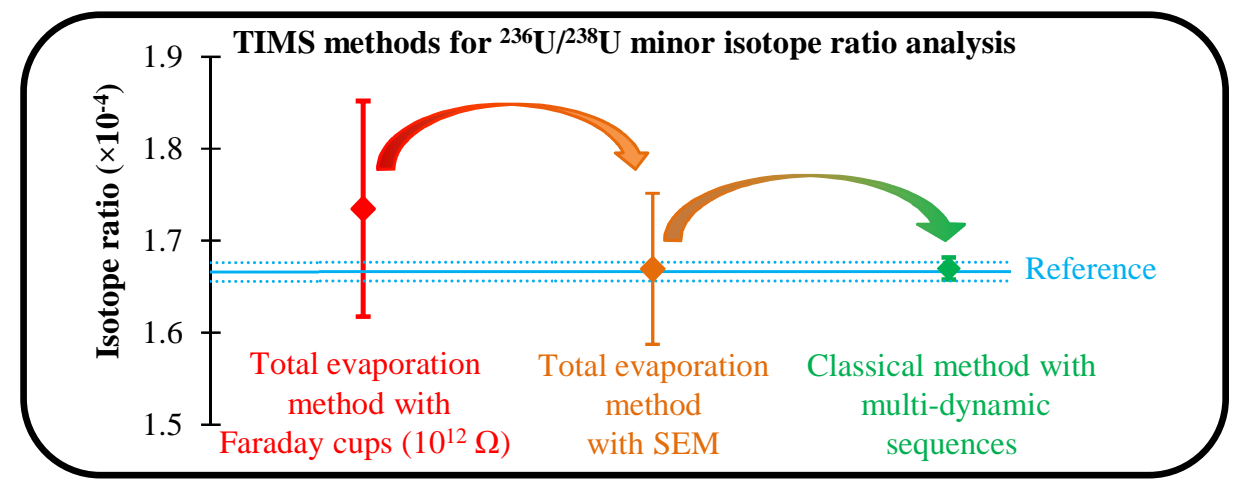

